\title{
Chorioretinal Biopsy-Proven Ocular Sarcoidosis in a Patient with a History of B-Cell Lymphoma
}

\author{
Sean T. Berkowitz ${ }^{a}$ Anderson L. Brock ${ }^{b}$ David A. Reichstein ${ }^{b}$

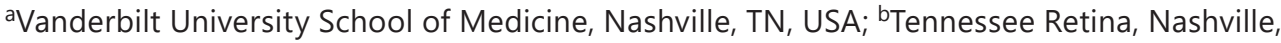 \\ TN, USA
}

\section{Keywords}

Ocular sarcoidosis $\cdot$ Chorioretinal biopsy $\cdot$ Noncaseating granuloma $\cdot$ Posterior uveitis

\begin{abstract}
The purpose of this report is to describe biopsy-proven ocular sarcoidosis (OS) in a 67-yearold patient with a history of sarcoidosis and diffuse large B-cell lymphoma (DLBCL). Nonspecific posterior chorioretinal lesions in a patient with prior malignancy necessitated chorioretinal biopsy to rule out metastatic lymphoma. The association between sarcoidosis and malignancy remains unclear and can complicate management of similar patients with nonspecific posterior segment findings. Chorioretinal biopsy may, therefore, be required to rule out malignancy in patients with a leading history.
\end{abstract}

\section{Introduction}

Sarcoidosis is a complex multisystem disease characterized by noncaseating granulomas described over one century ago. There are noteworthy racial and ethnic associations with an average annual incidence of 35.5 and 10.9 per 100,000 for black Americans and white Americans, respectively [1]. Ocular sarcoidosis (OS) as a manifestation of systemic sarcoidosis has varied prevalence estimates between 7 and 50\% [2]. Uveitis is the most common ocular manifestation, which can include anterior uveitis, posterior uveitis, or panuveitis with differential prevalence in specific racial groups [3].

The definitive diagnosis of OS is a biopsy of the lesion, which would show noncaseating granulomas; however, chorioretinal biopsy is infrequently performed due to potential ocular morbidity. In the absence of confirmatory biopsy, clinical criteria have been proposed by the International Workshop on OS. The criteria imply that infectious etiologies within the differential 
diagnosis have been ruled out. These criteria were revised in 2017 as a diagnosis of exclusion, with 7 intraocular clinical signs (keratic precipitates and/or iris nodules, trabecular meshwork nodules and/or anterior synechia, snowball vitreous opacities, multiple chorioretinal peripheral lesions, nodular and/or segmental periphlebitis and/or microaneurysm of an inflamed eye, optic-disc nodules/granulomas, and bilaterality) and 8 systemic investigation results (bilateral hilar lymphadenopathy (BHL), negative tuberculin test or interferon-gamma assays, elevated serum ACE, elevated serum lysozyme, elevated CD4/CD8 ratio (>3.5) in bronchoalveolar lavage, abnormal scintigraphy or positron emission tomography imaging, lymphopenia, or parenchymal lung changes). The new schema includes a definite diagnosis of OS based on positive biopsy and uveitis, presumed OS based on BHL and 2 intraocular signs, and probable OS if no biopsy evidence or BHL, but 3 intraocular signs and 2 systemic results are suggestive [4].

Posterior uveitis represents a broad group of diseases with infectious and noninfectious etiologies that can present similarly to nonuveitic masquerade syndromes. Ocular non-Hodgkin's lymphoma is a rare, difficult diagnosis and can classically masquerade as uveitis unresponsive to treatment. In older individuals, primary vitreoretinal lymphoma can masquerade as chronic posterior uveitis with multifocal fundus lesions that are initially responsive to tumoricidal steroid therapy. Intraocular tumors can either arise from the central nervous system/retina (primary CNS lymphoma or PCNSL) or spread hematogenously to the uvea (non-PCNSL or secondary intraocular lymphoma). PCNSL is rare and non-PCNSL is even more uncommon. Secondary intraocular lymphoma typically is confined to the choroid and arises most commonly from diffuse large B-cell lymphoma (DLBCL) [5]. These patients are more likely to have systemic symptoms and can present with anterior uveitis [6], hypopyon, or periorbital swelling; however, rare presentations such as panuveitis with serpiginous choroidopathy have been reported [7]. Early postmortem studies in patients dying of cancer suggest ocular metastasis occurs in roughly $6.7 \%$ of patients with systemic lymphoma [8]. Despite the rarity of non-PCNSL, the prior history of DLBCL as well as sarcoidosis in a patient presenting with new, diffuse bilateral chorioretinal lesions can create a diagnostic dilemma.

\section{Case Report}

A 67-year-old Caucasian man presented with a 1- to 2-year history of floaters in his left eye with associated blurry vision bilaterally. He denied eye pain, flashes, or floaters in the right eye. He denied fevers, night sweats, weight loss and did not demonstrate enlarged lymph nodes or respiratory symptoms. He had a complex past medical history, including a diagnosis of sarcoidosis several decades prior, hypothyroidism, as well as a complex cardiac history with 3 prior episodes of myocardial infarction treated with 2 stents and a quadruple bypass. Nine years prior, he was diagnosed with stage IV non-Hodgkin's lymphoma, DLBCL, involving the left anterior chest well, managed with 8 cycles of rituximab plus CHOP (rituximab, cyclophosphamide, doxorubicin, vincristine, and prednisone). His lymphoma was in remission for nearly 10 years prior to presentation.

On presentation, intraocular pressure was $16 \mathrm{~mm} \mathrm{Hg}$ in the right eye and $17 \mathrm{~mm} \mathrm{Hg}$ in the left eye. Best-corrected distance visual acuity (BCVA) was 20/40 - 1 in the right eye. BCVA in the left eye was $20 / 40+2$. Anterior segment exam demonstrated no stigmata or nodules consistent with sarcoidosis in either eye. The posterior segment exam was notable for clear vitreous bilaterally with multiple circumscribed deep yellow chorioretinal lesions bilaterally in the macula and periphery (Fig. 1). There was no associated subretinal fluid or macular edema.

Fluorescein angiography showed late staining of subretinal lesions consistent with drusen. Indocyanine green angiography showed choroidal blockage of parafoveal lesions.

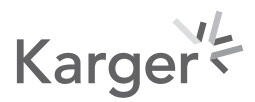



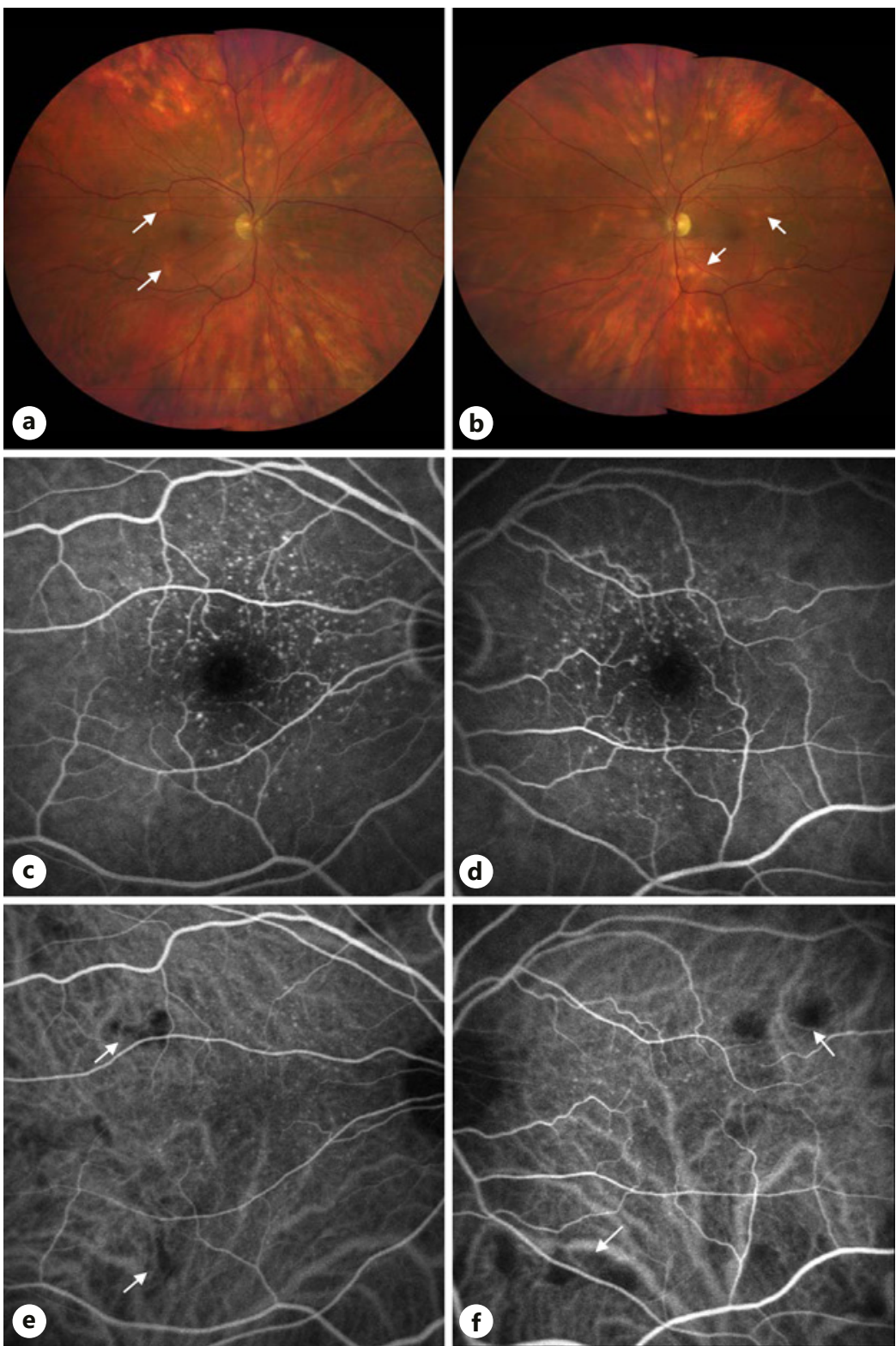

Fig. 1. Compilation of clinical images. a, b Right eye (a) and left eye (b) showing circumscribed yellow subretinal lesions bilaterally. $\mathbf{c}, \mathbf{d}$ Late phase fluorescein angiography showing late staining of the fovea consistent with drusen. e, f Early mid-phase indocyanine green angiography showing blockage of parafoveal lesions consistent with the fundus lesions in $\mathbf{a}, \mathbf{b}$ (arrows).

There was early blocked autofluorescence bilaterally. Serologic testing was significant for an unremarkable complete blood count, fluorescent treponemal antibody absorption nonreactive, QuantiFERON negative, rapid plasma reagin nonreactive, HIV 1,2 Ag/Ab nonreactive, and Lyme disease IgG negative. MRI of the brain and orbits with contrast was unremarkable. Chest, abdominal, and pelvic CT with intravenous contrast demonstrated extensive pulmonary reticulonodular thickening. PET imaging revealed no area suspicious for hypermetabolic tumor. After thorough discussion of the risks and benefits of observation and biopsy of the lesions, the patient elected for a biopsy of the right eye. 
25-gauge pars plana vitrectomy with chorioretinal biopsy was performed in the right eye. Briefly, after peritomy, 25-gauge ports were placed inferotemporally, superotemporally, and superonasally, and a chandelier was placed inferonasally. Following adequate vitrectomy with removal of the posterior hyaloid membrane out to the periphery, endodiathermy was used to mark the area for biopsy. A bimanual technique using a combination of internal limiting membrane forceps as well as vertical then horizontal scissors were used to excise the combined choroid and retina from the underlying sclera. Perfluorocarbon was injected to ensure that the macula would not detach during the chorioretinal tissue removal. Perfluorocarbon was then injected to float the biopsy tissue anteriorly in the vitreous. The superonasal sclerotomy was enlarged to remove the biopsy and subsequently closed with 8-0 vicryl suture. The internal limiting membrane forceps were used in the surgeon's left hand to feed the tissue to the surgeon's right hand. The tissue was then removed in 1 piece with a McPherson forceps. The specimen was sent half in formalin for permanent examination and half in RPMI for flow cytometry. Endolaser was used to surround the chorioretinal biopsy location. Perfluorocarbon was changed for air then subsequently changed for 1,000 Centistoke silicone oil. The sclerotomies and peritomies were sutured with 8-0 vicryl and 6-0 plain suture, respectively.

Flow cytometry of the specimen showed a hypocellular specimen with no CD19-positive or CD20-positive B cells and no immunophenotypically abnormal T-cells. A CD45-negative non-hematopoietic cell population was detected. Gross biopsies showed noncaseating granulomata, negative for atypical lymphoid infiltrate and malignancy, shown in Figure 2. Stains for periodic acid-Schiff, Grocott's methenamine silver, and acid-fast bacilli were performed. No fungal organisms or acid-fast bacilli were identified. The patient was diagnosed with OS and treated with topical prednisolone forte, atropine sulfate, and dexamethasone intravitreal implants in both eyes, with subsequent improvement in the lesions, bilaterally (Fig. 3). Fourteen months after the initial presentation, the patient's best-corrected distance visual acuity was 20/50 - 1 in the right eye and 20/30 - 2 in the left eye.

\section{Discussion}

This patient's bilateral chorioretinal lesions as well as prior systemic diagnosis of sarcoidosis would qualify as presumed OS by the revised International Workshop on OS criteria [4], but a competing cause for granulomatous uveitis such as metastatic lymphoma was present. Distinguishing features of OS include bilaterality, snowballs, mutton-fat keratic precipitates and/or iris nodules, and multiple chorioretinal lesions [9]. Sarcoidosis is significantly more prevalent than malignancy or other etiologies for de novo uveitis [10]; however, the risk of metastatic lymphoma in this patient with known prior DLBCL complicated diagnosis and management. New-onset uveitis in older patients is uncommon and worrisome, especially so in a patient with a prior malignancy and without characteristic anterior uveitic or granulomatous findings.

Vitreous biopsy has variable diagnostic yield, which may be improved with pars plana vitrectomy [5] with an estimated negative predictive value for lymphoma of $60.9 \%$ [11]. Often, vitreous biopsy is nondiagnostic requiring more invasive approaches. Chorioretinal biopsy is an effective means of diagnosing or ruling out lymphoma, which may be more effective in patients with marked vitritis [12]. While advances in vitrectomy technique and testing have increased the diagnostic yield, this patient had minimal vitritis and was suspected to have metastatic DLBCL which is typically confined to the choroid. These characteristics make chorioretinal biopsy a more pragmatic approach.

Interestingly, this patient received R-CHOP (rituximab, cyclophosphamide, doxorubicin, vincristine, and prednisone) for DLBCL. The influence of the immunomodulatory cascades of

\section{Karger'}



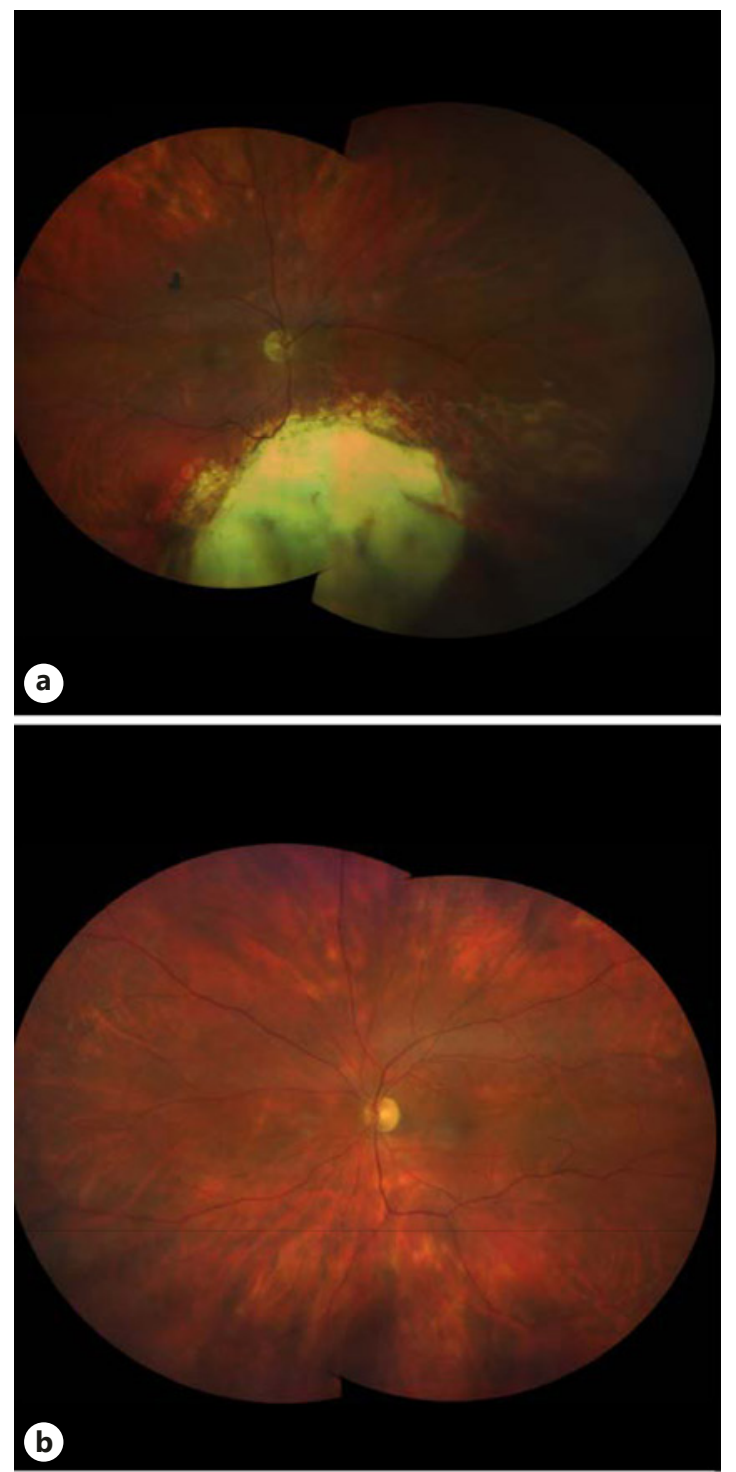

Fig. 2. Compilation of clinical images. a, b Posttreatment fundus photos of the biopsied right eye (a) and left eye (b) showing resolving chorioretinal lesions bilaterally. c Chorioretinal biopsy showing noncaseating granuloma (arrow) with multinucleated giant cells (arrowheads) with hematoxylin and eosin.

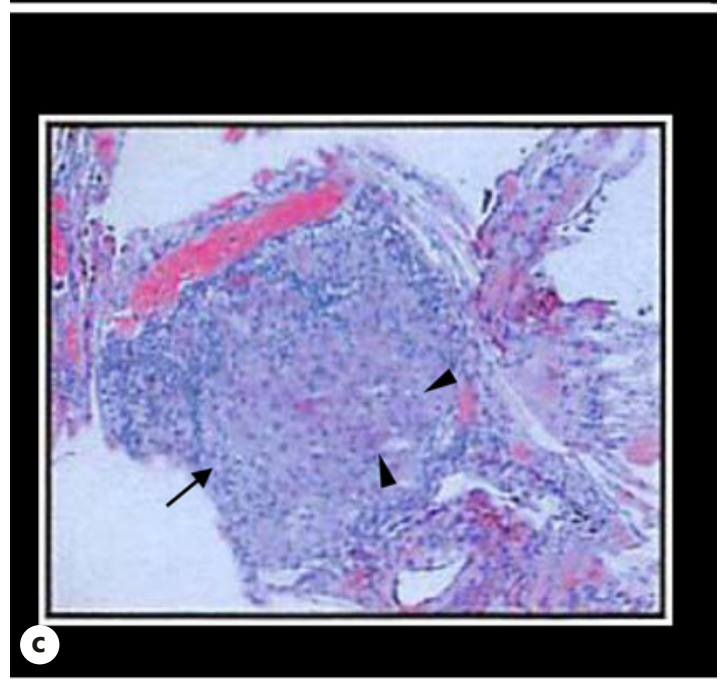



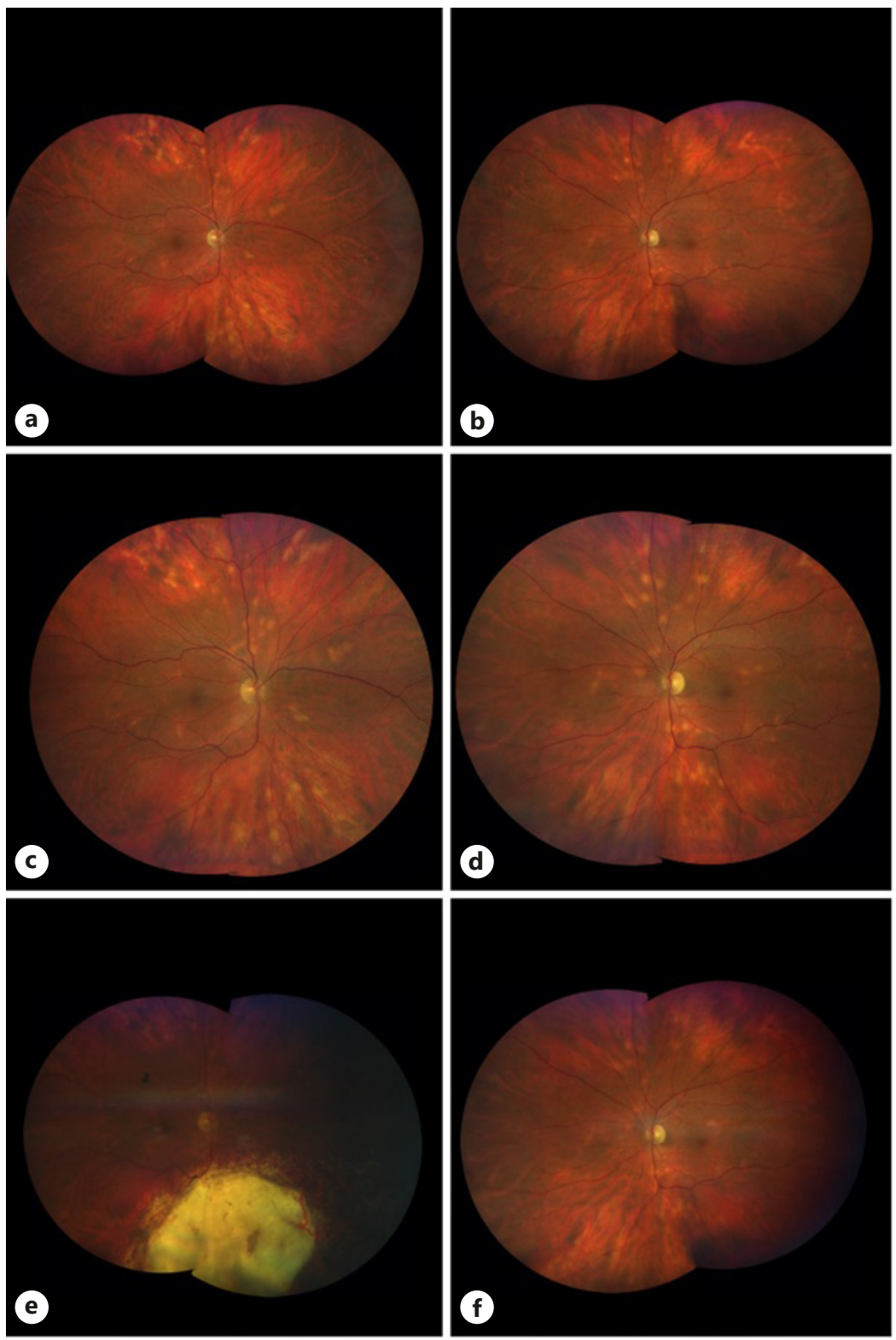

Fig. 3. Compilation of clinical images. a, b Right eye (a) and left eye (b) showing circumscribed yellow subretinal lesions bilaterally on presentation, 11 months later $(\mathbf{c}, \mathbf{d})$, and 18 months after $(\mathbf{e}, \mathbf{f})$ presentation, with regression of the lesions after topical prednisolone and dexamethasone intravitreal implants, bilaterally.

these chemotherapeutics on this patient's OS presentation is complex and unclear given systemic immunosuppressive and biologic agents are typically second-line agents for ocular sarcoidosis.

A case series of 9 patients diagnosed with primary intraocular lymphoma was later proven to have OS [13]. This is further complicated by the controversial sarcoid-lymphoma syndrome, with early associations suggesting sarcoidosis may cause immune system dysregulation that predisposes the immune system to develop lymphoma [14]. Multiple associations exist between sarcoidosis and hematologic or solid tumor malignancy, and sarcoidosis has been observed as both a paraneoplastic syndrome and in association with antineoplastic treatment [15]. 
Berkowitz et al.: Ocular Sarcoidosis and Systemic B-Cell Lymphoma

\section{Conclusions}

In patients with new-onset uveitis, chorioretinal biopsy may be required to differentiate sarcoidosis from worrisome masquerade syndromes. Here, a patient with a prior history of DLBCL, without obvious signs of granulomatous uveitis, was found to have OS on biopsy. Further refinement of diagnostic and management techniques may facilitate less invasive approaches for future patients.

\section{Statement of Ethics}

The patient consented to publication of the case orally and via written consent. This report does not contain any personal information that could lead to the identification of the patient.

\section{Conflict of Interest Statement}

The following authors have no conflicts of interest to declare: S.T.B., A.L.B., and D.A.R.

\section{Funding Sources}

No funding or grant support.

\section{Author Contributions}

All the authors attest that they meet the current ICMJE criteria for authorship. Substantial contributions to design, acquisition of data, and analysis: S.T.B., A.L.B., and D.A.R. Manuscript drafting and revising: S.T.B., A.L.B., and D.A.R. Final approval of submission: S.T.B., A.L.B., and D.A.R.

\section{References}

1 Rybicki BA, Major M, Popovich J, Maliarik MJ, Iannuzzi MC. Racial differences in sarcoidosis incidence: a 5-years study in a health maintenance organization. Am J Epidemiol. 1997 Feb;145(3):234-41.

2 Ungprasert P, Tooley AA, Crowson CS, Matteson EL, Smith WM. Clinical characteristics of ocular sarcoidosis: a population-based study 1976-2013. Ocul Immunol Inflamm. 2019;27(3):389-95.

3 Evans M, Sharma O, LaBree L, Smith RE, Rao NA. Differences in clinical findings between Caucasians and African Americans with biopsy-proven sarcoidosis. Ophthalmology. 2007 Feb;114(2):325-33.

4 Mochizuki M, Smith JR, Takase H, Kaburaki T, Acharya NR, Rao NA, et al. Revised criteria of International Workshop on Ocular Sarcoidosis (IWOS) for the diagnosis of ocular sarcoidosis. Br J Ophthalmol. 2019 Oct; 103(10):1418-22.

5 Reichstein D. Primary vitreoretinal lymphoma: an update on pathogenesis, diagnosis and treatment. Curr Opin Ophthalmol. 2016 May;27(3):177-84.

6 Tang LJ, Gu CL, Zhang P. Intraocular lymphoma. Int J Ophthalmol. 2017;10(8):1301-7.

7 Rattray KM, Cole MD, Smith SR. Systemic non-Hodgkin's lymphoma presenting as a serpiginous choroidopathy: report of a case and review of the literature. Eye. 2000 Oct;14(Pt 5):706-10.

8 Nelson CC, Hertzberg BS, Klintworth GK. A histopathologic study of 716 unselected eyes in patients with cancer at the time of death. Am J Ophthalmol. 1983 Jun;95(6):788-93.

9 Acharya NR, Browne EN, Rao N, Mochizuki M, Group IOSW. Distinguishing features of ocular sarcoidosis in an International Cohort of Uveitis Patients. Ophthalmology. 2018 Jan;125(1):119-26. 
10 Grumet P, Kerever S, Gilbert T, Kodjikian L, Gerfaud-Valentin M, De Parisot A, et al. Clinical and etiologic characteristics of de novo uveitis in patients aged 60 years and above: experience of a French tertiary center. Graefes Arch Clin Exp Ophthalmol. 2019 Sep;257(9):1971-9.

11 Davis JL, Miller DM, Ruiz P. Diagnostic testing of vitrectomy specimens. Am J Ophthalmol. 2005 Nov;140(5): 822-9.

12 Mastropasqua R, Thaung C, Pavesio C, Lightman S, Westcott M, Okhravi N, et al. The role of chorioretinal biopsy in the diagnosis of intraocular lymphoma. Am J Ophthalmol. 2015 Dec;160(6):1127-e1.

13 Birnbaum AD, Huang W, Sahin O, Tessler HH, Goldstein DA. Ocular sarcoidosis misdiagnosed as primary intraocular lymphoma. Retina. $2010 \mathrm{Feb}$;30(2):310-6.

14 Karakantza M, Matutes E, MacLennan K, O'Connor NT, Srivastava PC, Catovsky D. Association between sarcoidosis and lymphoma revisited. J Clin Pathol. 1996 Mar;49(3):208-12.

15 Cohen PR, Kurzrock R. Sarcoidosis and malignancy. Clin Dermatol. 2007 May-Jun;25(3):326-33. 